\title{
Colorectal Cancer Surgery in Elderly Patients
}

\author{
Young Jin Kim \\ Department of Surgery, Chonnam National University Hwasun Hospital, Chonnam National University Medical School, Hwasun, Korea
}

\section{See Article on Page 134-138}

Colorectal cancer is the second and the third most common cause of death from neoplastic disease in men and women, respectively, of all ages globally and is the fourth cause of death among cancers in Korea. Because life expectancy is increasing, the number of operations being performed on more elderly patients is also increasing. Elderly patients are more likely to have cardiovascular and pulmonary comorbidities that are associated with increased perioperative risk. Further, they tend to present with more locally advanced disease and are more likely to have an obstruction or disseminated disease. Mortality and morbidity after colorectal surgery in elderly patients are known to be high, with contributing factors being increased age, male sex, increased number of comorbidities, advanced disease, and postoperative medical and surgical complications [1]. Elderly patients with stage IV disease at the time of surgery, with comorbidities, and with postoperative complications are at risk for mortality during the first year after surgery [2]. However, several reports have shown that a colorectal-cancer resection in elderly patients is associated with neither higher postoperative mortality, complications, nor worse shortterm oncological outcome $[3,4]$. The short-term results, such as the 30-day morbidity and mortality, and the oncologic results, such as the 5-year survival rate, in elderly patients older than 85 years of age were similar to those in younger patients $[5,6]$.

Recently, laparoscopic surgery has been used for colorectal-cancer surgery. However, many colorectal surgeons are reluctant to perform laparoscopic surgery in older patients, given the vulnerable cardiopulmonary compliance, unstable perioperative hemodynamics, hypercapnia during longer operation times with expo-

Correspondence to: Young Jin Kim, M.D.

Department of Surgery, Chonnam National University Hwasun Hospital, Chonnam National University Medical School, 322 Seoyang-ro, Hwasun 58128, Korea

Tel: +82-61-379-7642, Fax: +82-61-379-7661

E-mail: kimyjin@jnu.ac.kr

(C) 2017 The Korean Society of Coloproctology

This is an open-access article distributed under the terms of the Creative Commons Attribution NonCommercial License (http://creativecommons.org/licenses/by-nc/4.0) which permits unrestricted noncommercial use, distribution, and reproduction in any medium, provided the original work is properly cited. sure to the risk of $\mathrm{CO}_{2}$ pneumoperitoneum, and the steep Trendelenburg position. Antoniou et al. [7], in their meta-analysis of 66,483 patients, reported a substantial benefit for elderly patients undergoing laparoscopic surgery in comparison with open colorectal surgery. An elective laparoscopic resection for colorectal cancer in the over 85-year age group is feasible and safe and offers advantages over open surgery [8,9]. Lim et al. [5] reported that the group of laparoscopically resected colorectal-cancer patients over 80 years of age had a significantly higher American Society of Anesthesiologists physical status classification, more preoperative comorbidities, and more advanced TNM stage than the group of patients younger than 60 years of age. However, neither the postoperative length of hospital stay nor the postoperative surgical and medical morbidities were significantly different between the 2 groups; neither was the disease-free survival rate $[10,11]$. Seishima et al. [12] reported that compared to open surgery, laparoscopic surgery was significantly associated with a decreased risk of perioperative mortality and postoperative complications.

Sex, the carcinoembryonic antigen concentration, and adjuvant therapy were significantly associated with overall survival in elderly patients with stage III colorectal cancer. The disease-specific and the disease-free survival rates for patients after an elective radical resection for Union for International Cancer Controlstage I-III cancer did not show significant differences related to age. Although early adjuvant chemotherapy can improve the survival of patients with advanced colorectal cancer, among patients 75 years of age or older with stage III CRC in Japan [13], 35\% with colon cancer and $21 \%$ with rectal cancer received adjuvant therapy.

The outcomes of colorectal cancer surgery in geriatric patients could be comparable to those in younger patients without increasing the postoperative morbidity and mortality rates. Moreover, laparoscopic surgery can reduce the length of hospital stay, the time to return to normal bowel function, and the rates of morbidities such as postoperative pneumonia, wound infection, and ileus. Adjuvant chemotherapy for elderly, stage-III, colorectal cancer patients can increase the long-term survival. Based on these findings, surgeons have no reason to be reluctant to perform either open or laparoscopic radical surgery for the treatment of patients with colorectal cancer, and adjuvant chemotherapy 
should be considered fort geriatric, colorectal-cancer patients.

\section{CONFLICT OF INTEREST}

No potential conflict of interest relevant to this article was reported.

\section{REFERENCES}

1. Devon KM, Vergara-Fernandez O, Victor JC, McLeod RS. Colorectal cancer surgery in elderly patients: presentation, treatment, and outcomes. Dis Colon Rectum 2009;52:1272-7.

2. Kornmann VN, van Vugt JL, Smits AB, van Ramshorst B, Boerma $D$. The first year after colorectal surgery in the elderly. Ann Coloproctol 2017;33:134-8.

3. Yen C, Simillis C, Choudhry M, Mills S, Warren O, Tekkis PP, et al. A comparative study of short-term outcomes of colorectal cancer surgery in the elderly population. Acta Chir Belg 2017 May 11:1-5 [Epub]. https://doi.org/10.1080/00015458.2017.1321269.

4. Basso SM, Lumachi F, Pianon P, Fanti G, Maffeis F, Ubiali P. Analysis of factors affecting short-term results in elderly patients undergoing elective surgical resection for stage I-II colon cancer. Anticancer Res 2017;37:1971-4.

5. Lim SW, Kim YJ, Kim HR. Laparoscopic surgery for colorectal cancer in patients over 80 years of age: the morbidity outcomes. Ann Surg Treat Res 2017;92:423-8.

6. Kazama K, Aoyama T, Hayashi T, Yamada T, Numata M, Amano S, et al. Evaluation of short-term outcomes of laparoscopic-assisted surgery for colorectal cancer in elderly patients aged over 75 years old: a multi-institutional study (YSURG1401). BMC Surg 2017;
17:29.

7. Antoniou SA, Antoniou GA, Koch OO, Pointner R, Granderath FA. Laparoscopic colorectal surgery confers lower mortality in the elderly: a systematic review and meta-analysis of 66,483 patients. Surg Endosc 2015;29:322-33.

8. Devoto L, Celentano V, Cohen R, Khan J, Chand M. Colorectal cancer surgery in the very elderly patient: a systematic review of laparoscopic versus open colorectal resection. Int J Colorectal Dis 2017 Jun 30 [Epub]. https://doi.org/10.1007/s00384-017-2848-y.

9. Li Y, Wang S, Gao S, Yang C, Yang W, Guo S. Laparoscopic colorectal resection versus open colorectal resection in octogenarians: a systematic review and meta-analysis of safety and efficacy. Tech Coloproctol 2016;20:153-62.

10. Jung WB, Shin JY, Suh BJ. The short-term outcome and safety of laparoscopic colorectal cancer resection in very elderly patients. Korean J Gastroenterol 2017;69:291-7.

11. Hinoi T, Kawaguchi Y, Hattori M, Okajima M, Ohdan H, Yamamoto S, et al. Laparoscopic versus open surgery for colorectal cancer in elderly patients: a multicenter matched case-control study. Ann Surg Oncol 2015;22:2040-50.

12. Seishima R, Okabayashi K, Hasegawa H, Tsuruta M, Shigeta K, Matsui S, et al. Is laparoscopic colorectal surgery beneficial for elderly patients? A systematic review and meta-analysis. J Gastrointest Surg 2015;19:756-65.

13. Yamano T, Yamauchi S, Kimura K, Babaya A, Hamanaka M, Kobayashi $\mathrm{M}$, et al. Influence of age and comorbidity on prognosis and application of adjuvant chemotherapy in elderly Japanese patients with colorectal cancer: a retrospective multicentre study. Eur J Cancer 2017;81:90-101. 